\title{
Can Dark Matter Halos Change Shape? Examining the Adams Instability in Triaxial Systems
}

\author{
Evan Dowling, Dr. Eric Barnes \\ Department of Physics \\ University of Wisconsin-La Crosse
}

\begin{abstract}
A recently discovered instability causes non-spherical orbits within dark matter halos to exponentially diverge into more spherical orbits. The instability was studied in limited regimes within the Adams et al. (2007) work. This project has replicated and extended the ideas to a static potential system that is accurate to all distances away from the potential's center as well as found an easy visualization for the arising instability. A better understanding of dark matter's behavior will help explain dark matter's halo structure and mass distribution that surrounds galaxies.
\end{abstract}

\section{Introduction}

Over the last forty years, substantial evidence has been found that suggests the majority of the mass within the universe is not stored within planets, stars, and intergalactic dust, but rather is hidden in the form of mysterious dark matter. This material is appropriately named, due to its peculiar property of not interacting with light. In fact, in the simplest current picture of dark matter behavior, gravity is the only force dark matter feels. Galaxies, like the one we inhabit, are hypothesized to be enveloped in dark matter halos whose mass can outnumber the residing stars 10 to 100 times (e.g., Salpeter 1978; Rubin 1979; Sancisi \& Allen 1979). So, why did astrophysicists and astronomers come to the conclusion that there needs to be so much "dark" mass?

It turns out the answer doesn't lie in just one area, but several. Once astronomers looked up to the night sky and painstakingly measured the velocities of the constituent stars within observable galaxies, they noticed speeds larger than expected. The amount of visible mass could not account for the gravitational force needed to keep the galaxies bound together (e.g., Rubin \& Ford 1970). Similarly, large amounts of mass beyond that accounted for by visible matter is needed to account for a phenomenon called gravitational lensing. Due to mass's ability to curve space-time, light follows curved paths near massive objects. This "bending" is analogous to the impact of an optical lens on a beam of light. To match astronomers' lensing observations, more matter than can be directly seen is needed (Schechter \& Wambsganss 2004; Ferreras et al. 2005). Finally, analyses of recent observations of the cosmic background radiation coincide with predictions made if the total mass in the universe far exceeds the mass attributed to protons, neutrons, and electrons (Spergel et al. 2007).

In an effort to better understand a substance that seems to prefer being hidden, astrophysicists have relied on computer simulations. Tracking orbits of particles that represent dark matter through collisionless systems is intended to cut through the fog and discover the true behavior of dark matter and the galaxies it underlies. Extensive research has been done laying the foundation for computational methods to analyze orbital systems (e.g. deZeeuw \& Merritt 1983; Statler 1987; Holley-Bockelmann et al. 2001, 2002; Poon \& Merritt 2002; Terzic \& Sprague 2007). 
Even the earliest studies of orbits found hints of instabilities (Henon 1973). One of the most widely studied of these instabilities has become known as the radial orbit instability (Merritt \& Aguilar 1985; Barnes et al. 1986; Palmer \& Papaloizou et al. 1987). A short summary of this type of instability starts with a spherical system, hosting particles on radial orbits (ones that would pass through the center of the sphere). The instability grows as neighboring orbits tend to become aligned along a direction that has slightly more particles than others. In the end, a spherical system can be transformed into an ellipsoidal system.

The instability we are interested in studying is essentially the inverse of the radial orbit instability. Instead of facilitating the change from spherical to ellipsoidal, the Adams instability acts to remove orbits that support non-spherical shapes. This instability was first identified using static (unchanging) gravitational fields produced by simple, analytically-describable mass density distributions. We use the term "simple" in the sense that the mathematical descriptions were abridged from an assumption of nearness to the potential's center. Particles that are perturbed slightly from certain orbits exponentially diverge from paths taken by non-perturbed particles. With orbits undergoing the instability being deflected in random directions, one would expect the host system to become more spherical or, at least, have a limited range of nonsphericity.

During the award period, we explored and expanded on the results on orbital instabilities expressed in Adams et al. (2007). The first step in this research project was to duplicate the results by converting relevant mathematical representations into computer code and comparing simulations given appropriate initial conditions. After this initial step was completed, we wanted to extend the accuracy of the instability to the entire range of the potential. Since the analytic forces depended on the assumption of nearness to the center it caused the equations to become increasingly inaccurate as the position grew away from the center. In our work we investigated different methods in order to accurately account for the forces and potential at all distances away from the center.

\section{Simulation and Results}

This work was completed within the University of Wisconsin-La Crosse physics computer lab and was entirely based on computational methods and analyses. Our computational investigation can be broken into two main parts.

Part one involved using computer simulations to replicate the work that first identified the orbital instability in non-spherical systems. This was done by transcribing the mathematical expressions for gravitational forces and potential into computer code; in our investigation the programming language was chosen to be FORTRAN. In order to best conserve energy for orbital integration an adaptive-timestep, fifth-order Runge-Kutta scheme was used. This numerical method allowed us to conserve orbit energy to a specified tolerance $\left(\Delta \mathrm{E} / \mathrm{E}<10^{-5}\right)$. With this complete we built analysis code in a second language, IDL, to read the output and display the data in graphs of position vs. time or surface of sections. Surface of section graphs are plots of position vs. velocity with various sets of orbits that share a common energy. This is done by adjusting a particles initial velocity (kinetic energy) in relation to its distance from the 
potentials center (potential energy) to guarantee that all particles start with identical total energies. To signal the onset of instabilities, the graphs show how unstable orbits lead to exponentially increasing position vs. time curves (Figs. $2 \& 5$ ) and appear as swarms of unconnected points (Fig. 6) within surface of section plots.

Replication of the results from Adams et al. 2007 and confirmation of the instability shortly followed.

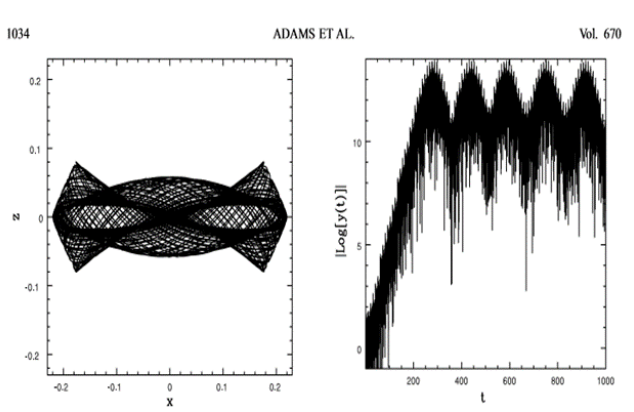

Figure 1. Graphs from Adams et al. Left: Orbit in principal x, z plane. Right: Development of instability with initial $y=10^{-8}$ (scaled distance).

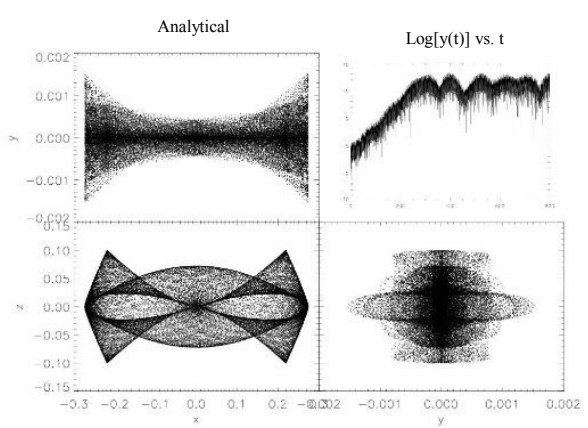

Figure 2. Orbit with nearly identical initial conditions as Fig 1. Panels show orbit shape in various axis planes. Top right: log plot showing exponential growth.

Looking at the left portion of Fig. 1 from Adams et al. and the bottom left graph of Fig. 2 the overall orbital shape within the $\mathrm{x}, \mathrm{z}$ plane is nearly identical. More importantly the logarithmic plots in both graphs show exponential growth in the y-position values meaning an instability, caused from the initial state, was present.

In order to better understand the instability, we sought for an easy visualization that might give grounds for its existence. When considering the limiting case of a particle traveling along one axis towards the center, we noticed that there was a discontinuous force behavior as it crosses the origin, like what is shown in Fig. 3. Once a particle crosses the origin it experiences a rapid, instantaneous force change. This "jerk" appears to assist in the instability of non-spherical orbits.

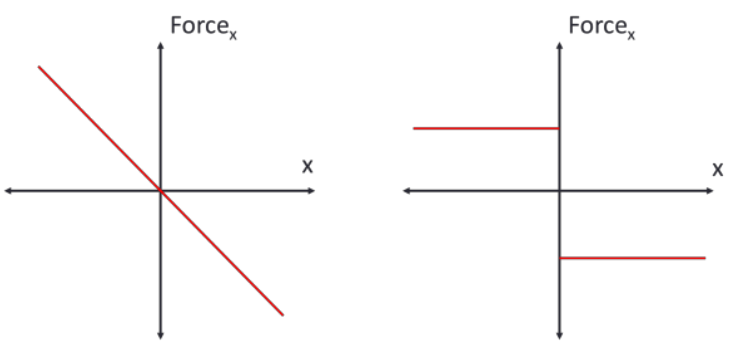

Figure 3. Left: $\mathrm{x}$-force behavior with non-zero $\mathrm{y}$ and $\mathrm{z}$ position. Right: $\mathrm{x}$-force behavior with $\mathrm{y}$ and $\mathrm{z}$ approximately zero with respect to $\mathrm{x}$.

Part two involved looking at a new system that allowed us to study the full potential accurately. This meant creating a new method to determine the forces and potential in contrast to the analytical descriptions used in part one. We went with numerically integrating the true expressions for potential and forces on a 3D grid. During an orbit, spline interpolation, another numerical method, was used to determine force values between grid points based on the perceived behavior of the grid. Using the same orbital integration Runge-Kutta method allowed for an acceptable energy conservation $\left(\Delta \mathrm{E} / \mathrm{E}<10^{-4}\right)$. Energy conservation was calculated by 


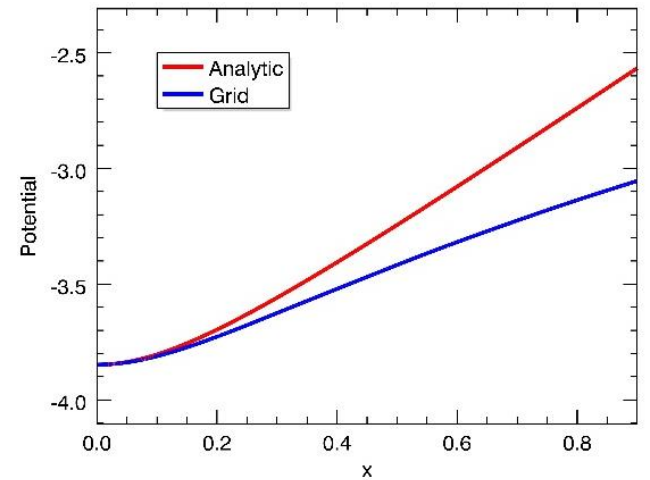

Figure 4. Differences between the analyticaland grid-based potentials. determining the spread in the energy values and dividing by the average energy magnitude. A low $\Delta \mathrm{E} / \mathrm{E}$ number signifies nearly constant energy values and energy conservation. The new grid-based description of the potential eliminated the error in values of force and potential since it doesn't rely on the approximation of nearness to the center. This is important since the grid-based potential deviates significantly from the analytical potential (correct only near the center) for larger radial distances, seen in figure 4.

Further examination of this new grid-based potential

has produced unstable orbits similar to those in Fig. 2 (Fig. 5) as well as evidence of the instability within a broader study of orbits seen in the inner fuzzy regions of the surfaces of section plot (Fig. 6).

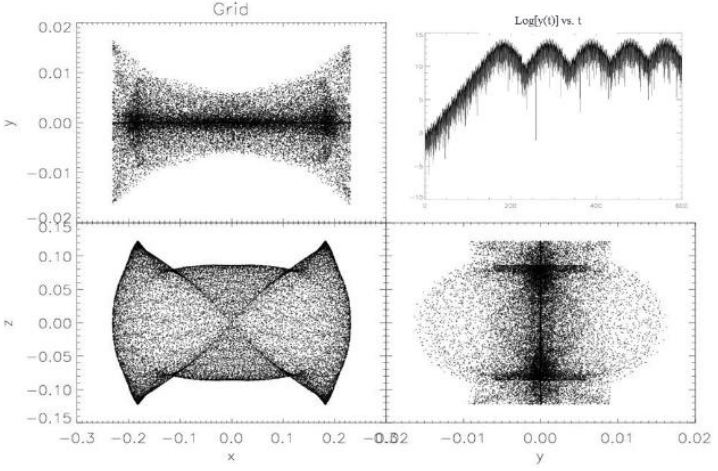

Figure 5. Top right: log plot showing exponential growth. Other plots show orbit shape in various axis planes.

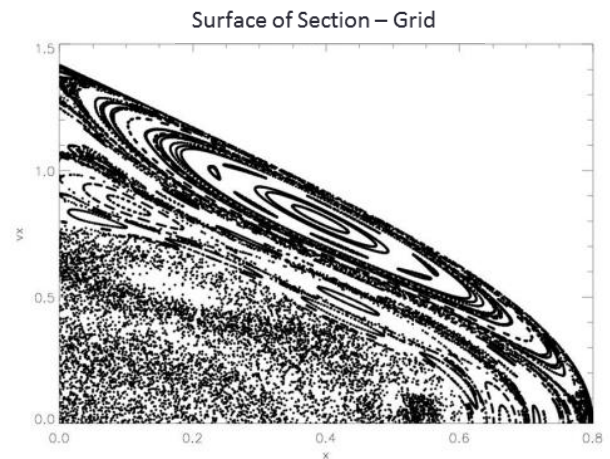

Figure 6. Surfaces of section of orbits with small initial y-position values.

For the sets of orbits within Fig. 6 the instability exists for x-position values as high as 0.6. The value 0.6 is a normalized distance of galactic dimension used to make the position variables dimensionless. When looking at Fig. 4 this instability exists even when the grid-based potential vastly differs from the analytical potential used in part one.

\section{Conclusion}

Through our investigation on the Adams instability we have verified past investigations as well as examined the instability in new ways. From reworking the initial investigation on the Adams instability, we developed the necessary analysis techniques to tackle new approaches on where the old investigation may be lacking. Also, by validating the instability with the analytical expressions we gained insight into an easy visualization on the source of the instability. From reforming the potential and forces into a 3D grid based on their true values we have overcome the inaccuracies that effected the initial investigation. This new approach also confirmed that instabilities exists throughout the entire potential and not just the inner regions where the previously studied analytical approximations were valid. 


\section{Future Work}

Our eventual goal is to search for evidence of the instability in $N$-body simulations, where there is no analytic description of the gravitational potential. With either positive or negative evidence, this investigation should merit publication in a peer-reviewed journal. These simulations involve many $(N)$ point particles evolving through time under their mutual gravitational attractions. Such a system has a "bumpy" potential due to discreteness effects particles are not smoothly distributed about every location in space. Adjusting for cosmological conditions, such as an expanding universe, we have begun creating simulations that will provide a very different testbed for comparison to the static potentials.

\section{References}

Adams, F., Bloch, A., Butler, S., 2007, ApJ, 670, 1027-1042.

Barnes, J., Hut, P., \& Goodman, J. 1986, ApJ, 300, 112.

de Zeeuw, T., \& Merritt, D. 1983, ApJ, 267, 571.

Ferreras, I., Saha, P., Williams, L. L. R. 2005, ApJ. Lett., 623, 5-8.

Henon, M. 1973, A\&A, 24, 229

Holley-Bockelmann, K., Mihos, J. C., Sigurdsson, S., \& Hernquist, L. 2001, ApJ, 549, 862.

Merritt, D., \& Aguilar, L. A. 1985, MNRAS, 217, 787.

Palmer, P. L., \& Papaloizou, J. C. P. 1987, MNRAS, 224, 1043.

Poon, M. Y., \& Merritt, D. 2001, ApJ, 549, 192 (PM01). 2002, ApJ, 568, L89.

Rubin V. C., Kent Ford W., 1970, ApJ, 159, 379

Rubin, V. C. 1979, Comments Astrophys., 8, 79-88.

Salpeter, E. E. 1978, in IAU Symp. 77, Structure and Properties of Nearby Galaxies, ed. E. M. Berkhuijsen \& R. Wielebinski (Dordrecht:Reidel), 23-26.

Sancisi, R., Allen, R. J. 1979, Astron. \& Astrophys., 74, 73-84.

Schechter, P. L., Wambsganss, J. 2004, in IAU Symp. 220, Dark Matter in Galaxies, ed. S. D. Ryder, D. J. Pisano, M. A. Walker, and K. C. Freeman, 103.

Spergel, D. N., Bean, R., Dore, 0., Nolta, M.R., Bennett, C.L., Dunkley, J., Hinshaw, G., Jarosik, N., Komatsu, E., Page, L., Peiris, H.B., Verde, L., Halpern, M., Hill, R.S., Kogut, A., Limon, M., Meyer, S.S., Odegard, N., Tucker, G.S., Weiland, J.L., Wollack, E., Wright, E.L. 2007, ApJ. Supp., 170, 377-408.

Statler, T. S. 1987, ApJ, 321, 113.

Terzic, B., \& Sprague, B. J. 2007, MNRAS, 377, 855. 